\section{Ultracold Molecules}

Françoise Masnou-Seeuws and Pierre Pillet

Laboratoire Aimé Cotton, Orsay, France

\section{Why cold molecules?}

Up to very recently, the coldest molecules in laboratory were at temperatures around $1 \mathrm{~K}$, either in supersonic beams, or on helium nanodroplets. The availability of even colder temperatures would open the route to many new applications. We may cite:

precision measurements: determination of lifetimes with possible estimation of parity non-conservation effects; measurement of an upper limit of the dipole moment of the electron as a critical test of elementary particle physics beyond the standard model [1]. Both in the case of atoms and molecules, the use of cold matter is improving the ultimate sensitivity of such measurements.

condensation of complex systems, for instance achievement of a molecular condensate. Many experimental and theoretical groups are presently working in that direction.

coherent control of molecule formation, towards ultracold chemistry.

interferometry with molecules [2].

For neutral molecules, major progress have been realized since 1997, along three main directions, using either non-optical or optical cooling techniques:

In the helium buffer gas cooling technique, developped in Harvard by John Doyle and collaborators, [3], hot molecules -for instance introduced by laser ablation- are thermalized with a cold helium buffer gas inside a dilution refrigerator, at temperatures well below $1 \mathrm{~K}$ (typically, $400 \mathrm{mK}$ ). This technique is related to the "sympathetic cooling" techniques at ultracold temperatures, widely used by the experimental groups working on Bose-Einstein condensation. The physical process of sympathetic cooling where a hot object is cooled via collisions with a cold one is easy to understand.

Another technique relies upon Stark deceleration of dipolar molecules in a supersonic beam. The basic idea of deceleration is to have a sequence of electrodes ( $\approx 100$ stages) making a static electric field that alternatively increases and decreases. Molecules in a specific rotational level are decelerated by the Stark effect when they propagate from a weak field to a strong one. The cycle is repeated, and each stage is removing kinetic energy from the beam equal to the increase in Stark shift. Although this idea has been known for many years, it was demonstrated only in 1999 by G. Meijer and his group: details on the method have been published recently [4]. The Stark deceleration technique is similar in principle to the acceleration techniques developed in particle accelerators, but of couse the size of the instruments is much smaller, less than $1 \mathrm{~m}$. Recently, a tabletop storage ring for molecules has been achieved. and temperatures below $1 \mathrm{mK}$ have been reached. Optical techniques are benefitting from the major progress in laser cooling and trapping of atoms. Direct laser cooling of molecules is not efficient, since it requires a large number of optical pumping cycles between the same two levels, whereas a molecule is a multi-level system. The solution [5-7] consists in first cooling the atoms with laser light, then making a molecule from two atoms via the photoassociation reaction. Next, the radiative dexcitation process must be guided to stabilize this excited molecule into a bound vibrational level of the ground electronic state. This technique is yielding molecules that are translationally cold $(\mathrm{T} \leq 100 \mu \mathrm{K})$, the population being spread into many vibrational levels. Further laser cooling should be implemented to obtain vibrationnally cold molecules.

We should also mention that groups working in ion traps are capable of cooling molecular ions down to $100 \mathrm{mK}$, using sympathetic cooling. In the following, we shall focus on optical cooling techniques, yielding ultracold molecules at temperatures below $100 \mu \mathrm{K}$.

\section{Making molecules via photoassociation of laser-cooled atoms and stabilization: mechanisms.}

In the photoassociation reaction [8] a pair of ultracold groundstate alkali atoms absorbs a photon, at frequency $v_{P A}=v_{0}-\Delta$, red-detuned relative to the resonance line,

$\mathrm{A}(n s)+\mathrm{A}(n s)+h\left(v_{P A}\right) \rightarrow \mathrm{A}_{2}^{*}\left(\Omega_{u, g}\left(n s+n p_{1 / 2,3 / 2}\right) ; v, J\right)$

creating a molecule in an excited electronic state $\Omega_{u, g}$. The corresponding potential displays an asymptotic $\sim R^{-3}$ behaviour, since at large distances an excited atom and a ground state atom interact via dipole-dipole interaction. This potential extends to much larger distances than the $\sim R^{-6}$ ground state potential. The reaction is efficient at low detunings $\Delta$, forming molecules in very excited vibrational levels $v$. In contrast the rotational quantum number J remains small due to the very low kinetic energy of the colliding atoms in the initial state which limits the reaction to small centrifugal barriers. The photoassociated molecule is a long range molecule, which looks like a pair of atoms at very large distances more than a usual molecule. Indeed, during a vibration period, the relative motion is taking place most of the time in the

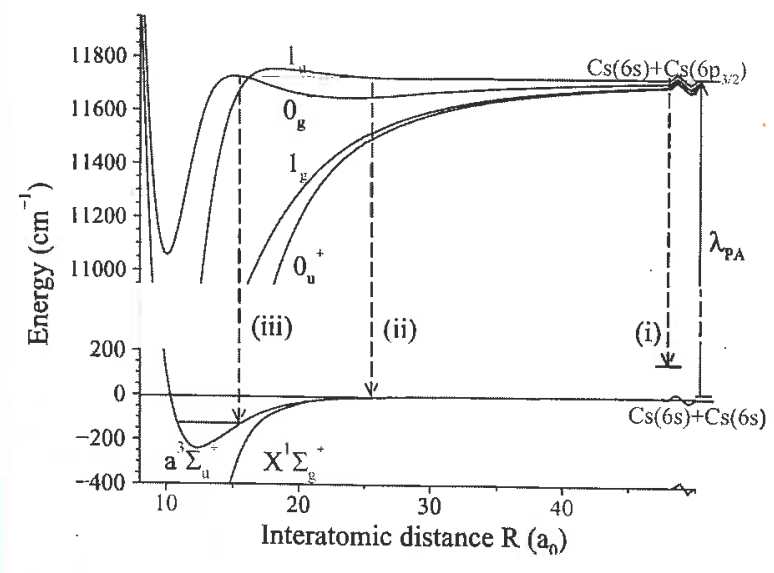

4. Fig. 1: Formation of an excited molecule by photoassociation of two cesium ground state atoms. (i) In most cases, this shortlived molecule decays back into a pair of cold atoms. Spontaneous emission $\rightarrow$ dissociation.(ii) $1_{u}$ "speed bump" $\rightarrow$ stabilization by spontaneous emission to a bound level of the ground state.(iii) $0_{\bar{g}}^{-}$ "speed bump" $\rightarrow$ stabilization by spontaneous emission to a bound level of the lower triplet state. 
asymptotic region governed by the very weak $R^{-3}$ potential, the time spent in the inner region being comparatively very short. At small distance, the relative motion is accelerated by the chemical forces and reflected on the steep potential wall. Therefore when this excited molecule decays by spontaneous emission, it is likely to dissociate into a pair of ground state cold atoms. Stabilization by spontaneous emission into a bound level of the ground electronic state requires very particular situations. The first scheme was found accidentally for $\mathrm{Cs}$ [5], and confirmed for $\mathrm{Rb}$ [7], and is schematized in Fig. 1. Due to the presence of a double well potential with a very gentle slope, the vibrational motion in the region of intermediate distances is delayed, so that there is a non negligible probability of stabilization by spontaneous emission into a bound level of the ground state.

This scheme is fortuitous, since for lighter alkali dimers the double well is located at even larger internuclear distances where decay into bound levels of the ground state is impossible. However, once we have understood that the key effect is to slow down the vibrational motion in the intermediate region, many other schemes can be found. For instance a resonant coupling scheme, demonstrated for $\mathrm{Cs}_{2} 0_{u}^{+}\left(\mathrm{P}_{1 / 2,3 / 2}\right)$ coupled channels [9], can be present in many molecules. Besides, schemes using induced emission could be very efficient, so this research domain is only opening.

\section{Formation and trapping of cold molecules: experiment}

Most of the experiments of photoassociation are performed by using the basic tool of any experiment with cold atoms: a magneto-optical (MOT). Figure 2 shows a schema of the experimental setup of a vapor-loaded cesium MOT. The atomic cloud is illuminated with a continuous-wave laser of frequency $v_{P A}$ to produce the resonant photoassociation reaction. In most of the photoassociation configurations considered experimentally, the electronically excited $\mathrm{Cs}_{2}^{*}$ molecules dissociate by giving a pair of atoms which escape outside of the trap (see arrow (i) in Fig. 1). Trap-losses can be analyzed by recording the fluorescence yield due to the cooling laser beams. Formation of cold ground-state molecules after emission of a photon with frequency $v_{S P}$, for instance

$$
\mathrm{Cs}_{2}^{*}\left(0_{\mathrm{g}}^{-}\left(6 s+6 p_{3 / 2}\right) ; v, J\right) \mathrm{Cs}_{2}\left(a^{3} \Sigma_{u^{+}}^{+} v^{\prime}, J^{\prime}\right)+h v_{S P},
$$

yields molecules in the lower triplet state (see arrow (iii) in Fig. 1). They can be detected by photoionization, using a pulsed dye laser beam. The $\mathrm{Cs}_{2}^{+}$molecular ions are then selectively detected through a time-of-flight mass spectrometer. The upper inset of

Fig. 2 shows a typical rotational progression obtained by scanning the frequency of Two hundred thousand the photoassociation laser around the resonance for molecules can be $\begin{aligned} & y=6 . \text { One important applica- } \\ & \text { tion of such spectra is }\end{aligned}$ accum accurate determination of the accumulated and excited potential, leading, through $C_{3}$ coefficient in the asymptotic $C_{3} / R^{3}$ expansion, to unprecedented accuracy in atomic lifetimes. In these experimental conditions, rates of formation can reach up 0.2 molecule per atom and per second, meaning several millions of molecules formed per second for an initial atomic cloud with several tens millions of atoms [10]. The cold molecules being unsensitive to the MOT trapping forces are falling down because of gravity. The analysis of their ballistic expansion allows to determine their temperature which is found identical to that of the atomic cloud, i.e. in the microkelvin range [5].

To accumulate and to store the formed cold molecules is a necessary condition for further applications. The use of a $\mathrm{CO}_{2}$ laser to create a quasi-electrostatic trap is an interesting possibility, already demonstrated for the molecules present in the MOT [11], and which should be confirmed for those obtained via photoassociation. Another way has been investigated, via a mixed atomic and molecular trap, constituted by a Cs vapor-cell MOT and a quadrupolar magnetic $\mathrm{Cs}_{2}$ trap, using the same magnetic field gradient. The atomic cloud is produced at the intersection of the three pairs of mutually othogonal, counter propagating $\sigma^{+}-\sigma^{-}$lasers beams, at the zero magnetic field point of a pair of anti-Helmholtz coils, but here the magnetic field gradient of $6 \mathrm{mT} / \mathrm{cm}$ is chosen four times larger than for an usual
A. Fig. 2: Experimental setup of the molecular trap. The upper inset shows a typical photoassociation spectrum (note the resolution), the lower one represents the spatial analyzis of the molecular cloud.

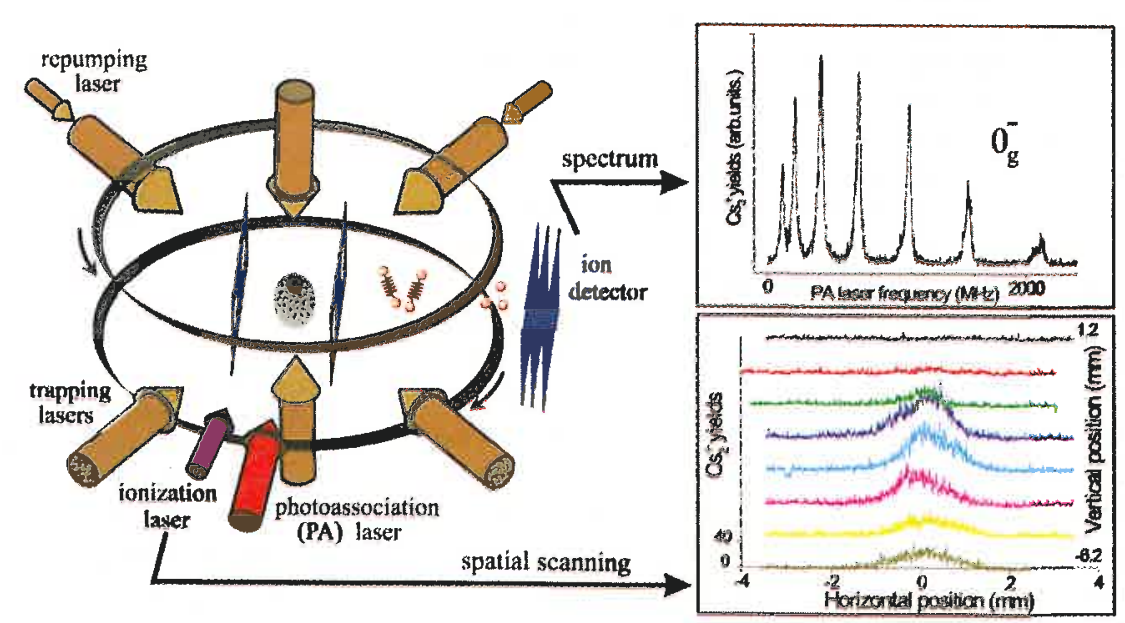


